\title{
Diseño robusto para optimizar pico sistemas solares en el Valle del Mantaro'
}

\section{Robust design to optimize peak solar systems in the Mantaro Valley}

Lazo Baltazar, B. / Huari Vila, O.

Facultad de Ingeniería Mecánica, Universidad Nacional del Centro del Perú

\section{RESUMEN}

El objetivo de la investigación es diseñar un sistema robusto para optimizar pico sistemas solares en el Valle del Mantaro. Para lo cual se ubicó el lugar de investigación en la cuadra doce del Jirón Libertad del distrito de Huancayo, 12 grados de latitud sur y longitud 75 grados sur. Como resultado se encontró que los factores más significativos en el sistema pico solar estudiado son: el ángulo de inclinación, la orientación, el tiempo de carga y la estación cuando se realiza las mediciones.

Palabras clave: Diseño robusto, sistemas solares.

\section{ABSTRACT}

The research objective is to design a robust system to optimize peak solar systems in the Mantaro Valley. For which the research site was located in the block Jiron Libertad twelve district of Huancayo, 12 degrees south latitude, longitude 75 degrees south. As a result it was found that the most significant peak in the solar system factors studied are: the angle of inclination, orientation, load time and season when measurements are performed.

Keywords: Robust design, solar systems. 


\section{INTRODUCCIÓN}

La energía solar fotovoltaica se basa en el efecto fotoeléctrico, es decir, en la conversión de la energía lumínica proveniente del sol en energía eléctrica. Para llevar a cabo esta conversión se utilizan unos dispositivos denominados células solares, constituidos por materiales semiconductores en los que artificialmente se ha creado un campo eléctrico constante. El material más utilizado es el Silicio Estas células conectadas en serie o paralelo forman un panel solar encargado de suministrar la tensión y la corriente que se ajuste a la demanda

En los últimos años se viene produciendo un aumento notable de instalaciones de energía fotovoltaica debido, por una parte, a la mayor sensibilidad social y política hacia temas medioambientales $y$, por otra, a la continua mejora y reducción de costes de los sistemas solares fotovoltaicos. El presente trabajo de investigación tiene el objetivo de diseñar un sistema robusto para optimizar un pico sistema solar del Valle del Mantaro, cuyo sustento científico se centra en la optimización del funcionamiento de los sistemas poco sensibles a diversos factores.

\section{MATERIALES Y MÉTODOS}

\section{Materiales}

La presente investigación se realiza usando principalmente en un pico sistema solar fotovoltaico de fabricación India, modelo Sun King Pro, con un panel solar de $2.5 \mathrm{Wp}$, que opera con un foco led de tres niveles de iluminación (100 lúmenes, 40 lúmenes y 20 lúmenes). Del mismo modo termómetros, medidores de viento, y software especializado de apoyo.

\section{Métodos}

El tipo de investigación es tecnológica, nivel de investigación de estudios de comprobación de hipótesis causales, método de investigación experimental.

\section{RESULTADOS}

Las variables estudiadas aplicando el Método Taguchi, definiendo los niveles de estudio, de manera indistinta cualitativos y cuantitativos.

Tabla 1. Factores de evaluación Taguchi

\begin{tabular}{clcc}
\hline CÓDIGO & FACTORES CONTROLABLES & NIVEL 1 & NIVEL 2 \\
\hline A & ORIENTACIÓN & OESTE & NORTE \\
B & ANGULO DE INCLINACIÓN & 0 & 12 \\
C & CLIMA & NUBLADO & SOLEADO \\
D & TIEMPO DE CARGA & 4 & 6 \\
E & HUMEDAD RELATIVA & ALTA & BAJA \\
F & VELOCIDAD DEL VIENTO & ALTA & BAJA \\
G & ESTACIÓN & PRIMAVERA & VERANO \\
\hline
\end{tabular}

Luego de hacer las mediciones en base a la matriz Taguchi L8, con tres réplicas.

Tabla 2. Matríz de evaluación Taguchi

\begin{tabular}{|c|c|c|c|c|c|c|c|c|c|}
\hline \multirow[t]{2}{*}{$A$} & \multirow[t]{2}{*}{ B } & \multirow[t]{2}{*}{ C } & \multirow[t]{2}{*}{ D } & \multirow[t]{2}{*}{$E$} & \multirow[t]{2}{*}{$\mathrm{F}$} & \multirow[t]{2}{*}{ G } & \multicolumn{3}{|c|}{$\begin{array}{l}\text { Tiempo llumina- } \\
\text { ción (horas) }\end{array}$} \\
\hline & & & & & & & R1 & R2 & R3 \\
\hline 1 & 1 & 1 & 1 & 1 & 1 & 1 & 4.5 & 5.5 & 4.8 \\
\hline 1 & 1 & 1 & 2 & 2 & 2 & 2 & 4.2 & 5.5 & 6.2 \\
\hline 1 & 2 & 2 & 1 & 1 & 2 & 2 & 6.1 & 6.5 & 6.8 \\
\hline 1 & 2 & 2 & 2 & 2 & 1 & 1 & 5.6 & 5.9 & 6.0 \\
\hline 2 & 1 & 2 & 1 & 2 & 1 & 2 & 7.1 & 6.5 & 6.8 \\
\hline 2 & 1 & 2 & 2 & 1 & 2 & 1 & 4.0 & 4.8 & 5.4 \\
\hline 2 & 2 & 1 & 1 & 2 & 2 & 1 & 7.9 & 8.2 & 7.8 \\
\hline 2 & 2 & 2 & 2 & 1 & 1 & 2 & 7.1 & 7.9 & 7.5 \\
\hline
\end{tabular}

Encontramos los siguientes resultados.

Tabla 3. Respuesta para relaciones de señal de ruido más grande es mejor.

\begin{tabular}{cccccccc}
\hline Nivel & A & B & C & D & E & F & G \\
\hline 1 & 14.85 & 14.46 & 15.85 & 16.15 & 15.19 & 15.80 & 15.10 \\
2 & 16.36 & 16.75 & 15.36 & 15.06 & 16.02 & 15.41 & 16.11 \\
Delta & 1.51 & 2.229 & 0.49 & 1.10 & 0.84 & 0.39 & 1.01 \\
Clasificar & 2 & 1 & 6 & 3 & 5 & 7 & 4 \\
\hline
\end{tabular}


Figura 1. Gráfica de efectos principales donde se puede notar los efectos en escala de significación en el sistema pico solar estudiado.

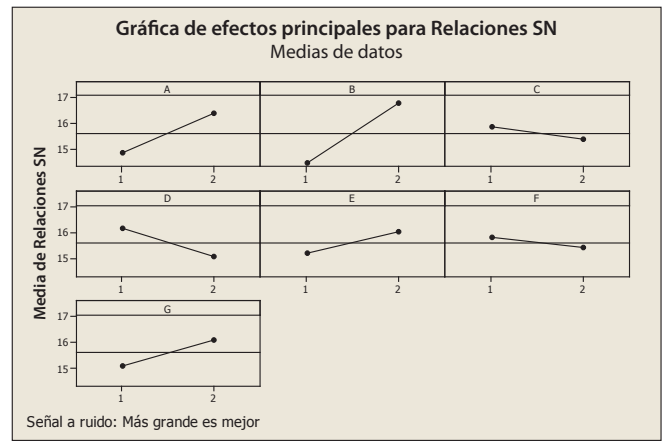

\section{DISCUSIÓN}

Los factores estudiados en orden de significación en el sistema estudiado se dan en el siguiente orden: (1 - B - ángulo de inclinación), (2 - A - orientación), (3 - D - Tiempo de carga), (4 - G - estación), (5 - E - humedad relativa), (6 - C - clima), (7 - F - velocidad de viento).

\section{CONCLUSIONES}

Los factores más significativos en el sistemas pico solar estudiados los factores más significativos son el ángulo de inclinación, la orientación, el tiempo de carga y la estación cuando se realiza las mediciones.

Los resultados confirman las exigencias del método F - chart.

\section{AGRADECIMIENTOS}

Nuestros agradecimientos a los colegas de la Facultad de Ingeniería Mecánica que apoyaron en la realización del presente trabajo.

\section{REFERENCIAS BIBLIOGRÁFICAS}

1. Gutiérrez H., De la Vara R. (2008). Análisis y diseño de experimentos. Editorial Mc Graw Hill. México.

2. Montgomery D. (2005). Diseño y análisis de experimentos. Editorial Prentice Hall. México.

3. Mc Cormac R. (2002). Manual de la ener- gía solar. Editorial Prentice Hall. México.

4. Ogata K. (2003). Ingeniería de control moderna. Editorial Prentice Hall. México.

5. Ostolaza H. (2004). Energía solar y sus aplicaciones. Editorial Prentice Hall. México.

6. Pérez G. (2002). Sistemas solares y sus aplicaciones. Editorial Mc Graw Hill. Méxic

7. Figura 1. Gráfica de efectos principales. 